\title{
A Case of Myoclonic Epilepsy Presenting with Status Epilepticus in an Elderly Male Patient
}

\author{
Halil Onder ${ }^{1}$ \\ ${ }^{1}$ Department of Neurology, Yozgat City Hospital, Yozgat, Turkey
}

\begin{abstract}
Address for correspondence Halil Onder, MD, Department of Neurology, Yozgat City Hospital, Yozgat 66000, Turkey (e-mail: halilnder@yahoo.com).
\end{abstract}

\begin{abstract}
Myoclonic epilepsy in the form of status epilepticus is an extremely rare reported presentation. Herein, we describe an 87-year old male patient presenting with abrupt-onset rhythmic myoclonic jerks that were evaluated as synchronous positive and negative myoclonus. Further etiological investigations revealed that the myoclonus was associ-

Keywords

- negative myoclonus

- status epilepticus

- stroke

- elderly

- pathophysiology ated with ictogenesis, and appropriate antiepileptic treatment provided total cessation of the movements. To my knowledge, this is a unique case of myoclonic status epilepticus in an elderly patient, which may have been associated with a cerebrovascular disease. In the presentation of this case, we will review the related literature and discuss some considerations to explain the pathophysiology of epileptic myoclonic movements and the possible role of pontine lesions.
\end{abstract}

\section{Introduction}

Epileptic negative myoclonus (ENM) is an uncommon seizure type characterized by a sudden, brief loss of muscle tone that may lead to falling. Most of the reported cases of idiopathic epilepsy with ENM were cases of benign childhood epilepsy with centrotemporal spikes (BCECTS). ${ }^{1}$ However, rarely, it has been reported in adult individuals. ${ }^{1}$ Although the underlying mechanisms remain to be elucidated, the pathophysiological hypotheses on the origin of myoclonus have been discussed in the setting of abnormalities with both subcortical and cortical sources. ${ }^{2,3}$ Nonetheless, there is a considerable amount of evidence supporting the hypothesis of cortical inhibitor activity as the mediating mechanism of ENM. ${ }^{4,5}$ Some authors believe that positive myoclonus is another aspect of the same phenomenon, resulting from an altered sensory motor cortex output producing different degrees of positive or negative effects on the motor system. ${ }^{6}$ Herein, we present an extremely rare elderly patient with both positive and negative myoclonus that was found to be epileptic in nature and discuss the possible pathophysiologies. Through the presentation of this uncommon case and a literature review, we will expand the clinical spectrum of isolated myoclonus.

\section{Case Report}

An 87-year-old-male patient was admitted to our clinic due to rhythmic myoclonic jerks which had started abruptly the previous night and continued up to admission. There was no history of a new-onset drug, infection, or head trauma. No history of any previous seizure was taken from the patient or his relatives. The family history was unremarkable. On neurological examination, the patient was orientated and cooperative, although the cooperation of the patient was mildly limited due to hearing loss caused by an ear infection in childhood. Remarkably, his relatives had noted no deterioration in his communication level. The results of other investigations, including motor and sensory, were within normal limits.

Brief shock-like jerks of the whole body, which were obvious in the upper extremity, were observed. They were characterized by rhythmic $(0.5-1 \mathrm{~Hz})$ and brief interruptions of muscular activity that were compatible with negative myoclonus. However, positive myoclonic movements were also recognized, which were prominent in the facial muscles (-Video 1); available online only). Moreover, the myoclonic jerks were also sustained during volitional activities, and there was no entrainment or distractibility. 


\section{Video 1}

Rhythmic (0.5-1 Hz), brief interruptions of muscular activity that was compatible with negative myoclonus. Also, positive myoclonic movements were also recognized, prominent in the facial muscles. Online content including video sequences viewable at: https://www.thiemeconnect.com/products/ejournals/html/10.1055/s-00401709348.

Myoclonic movements had no tactile and auditory sensitivity. Phenomenologically, the symptoms were evaluated as generalized synchronous positive and negative myoclonus prominent in the upper extremities.

Laboratory results, including a hemogram, serum biochemistry, vitamin B12, and folic acid levels, C-reactive protein, sedimentation, and complete urinalysis, were within normal limits. A cranial magnetic resonance imaging (MRI) showed chronic ischemic lesions in the bilateral pontine tegmentum, bilateral thalamic region, basal ganglia, and periventricular area (-Fig. 1). Notably, a routine electroencephalography (EEG) showed generalized discharges of high-voltage spike/polyspike-wave complexes continuing for more than half of the EEG recording (-Fig. 2A). However, the background activity was within normal limits ( $8 \mathrm{~Hz}$ ).

In view of the evidence, the diagnosis of late-onset myoclonic status epilepticus was established, and diazepam 5-mg intravenous (IV) was administered, which yielded the cessation of the myoclonic jerks. A maintenance treatment of valproic acid $2 \times 500 \mathrm{mg}$ daily was initiated afterward. The patient was hospitalized for clinical follow-up. A routine EEG performed the next

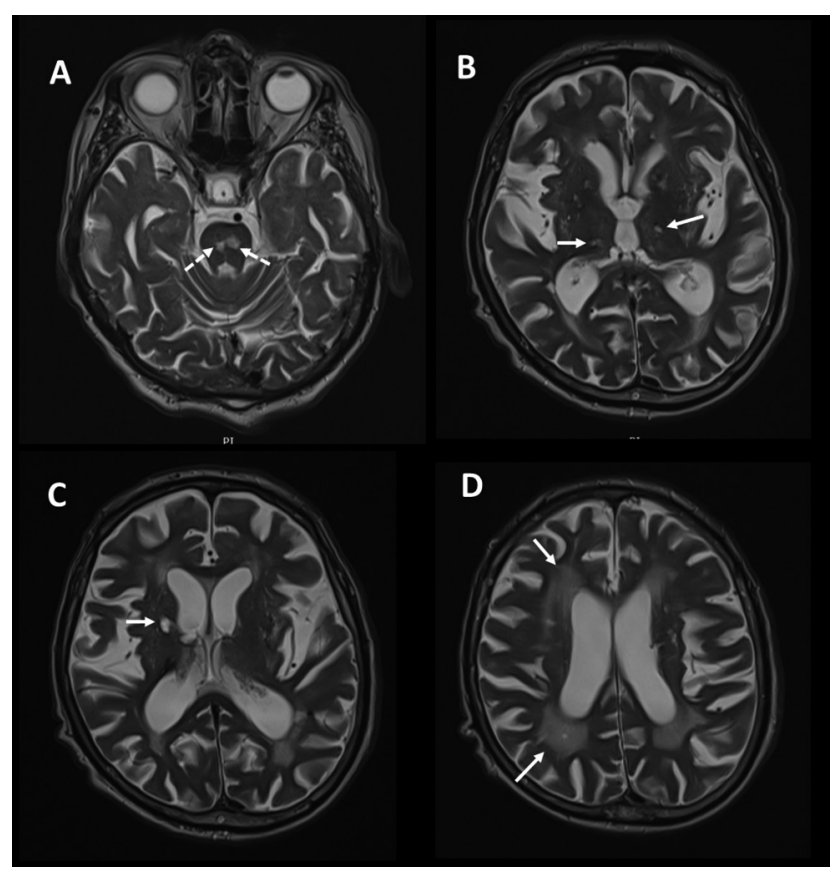

Fig. 1 Cranial axial T2-weighted images showing chronic ischemic lesions in the bilateral pons corresponding to the basis pontis which includes (A) bilateral corticospinal and corticobulbar tracts, (B) thalamus, (C) right globus pallidus, and (D) bilateral periventricular region (arrows). day showed near-total resolution of the epileptiform discharges ( - Fig. 2B), and the patient did not suffer from myoclonic movements in the follow-up ( - Video 2 ).

\section{Discussion}

Although epilepsy is mainly believed to be a disease of youth, it has been shown that nearly $25 \%$ of new-onset seizures occur after 65 years of age. ${ }^{7}$ The incidence in people over 80 years is triple its rate in children. ${ }^{8,9}$

However, the causes and clinical manifestations of seizures and epilepsy in the elderly differ from those in younger people. ${ }^{10}$ The most common causes of acute symptomatic seizures in elderly have been reported as cerebrovascular disease, metabolic disturbances, dementia, traumatic brain injury, tumors, and drugs. ${ }^{8,10,11}$ Among these, acute stroke is the most common cause, accounting for up to half of cases in older adults. ${ }^{12}$ Although its occurrence is particularly increased in the immediate poststroke period, the risk of epilepsy is also known to be significantly increased in the chronic phase, such that it was found to be 20 times higher in the first year after a stroke. ${ }^{13}$

In our patient, the cranial MRI did not reveal an acute ischemic lesion. However, extensive, chronic ischemic brain lesions were recognized, and no other etiological risk factors were identified. On the whole, it appears that the most likely etiological classification of epilepsy in our patient is epilepsy due to stroke. To our knowledge, status epilepticus presenting with negative myoclonus as the initial manifestation in an elderly patient has not been reported previously. Therefore, the illustration of this case is extremely important which may provide crucial perspectives regarding the underlying pathophysiology of myoclonus. In a unique report, synchronous positive and negative myoclonus due to pontine hemorrhage was defined in a patient by Palmer et al. ${ }^{14}$ However, the origin was not evaluated as ictogenic and antiepileptic treatment did not yield an amelioration as well, which was completely distinct in nature from our patient.

ENM can be defined as an interruption of tonic muscular activity, time-locked with a spike or sharp wave on EEG, without evidence of antecedent myoclonus. ${ }^{1}$ ENM, as an initial manifestation of epilepsy, has been reported in pediatric patients with BCECTS, previously. ${ }^{3}$ In an extremely rare report, an 18-year-old woman with ENM was described. ${ }^{1}$ In this patient, EEG showed almost continuous generalized spike-wave discharges as in our case. However, cranial MRI was normal and the authors preferred to define the etiologic category of myoclonus as idiopathic. ${ }^{1}$ On the other hand, perfusion imaging of the brain showed cerebral disturbances mainly in the parietal region contralateral to the limbs affected by ENM. ${ }^{1}$ In our case, there was no cortical lesion in the parietal lobe. However, we cannot exclude the possibility of an underlying parietal malfunction due to the limitation of that perfusion imaging and advanced electrophysiological investigations were not performed in this patient. Literature evidence draws attention to the involvement of the postcentral region in the genesis of ENM. This was supported by a study showing sustained negative myoclonus that was 


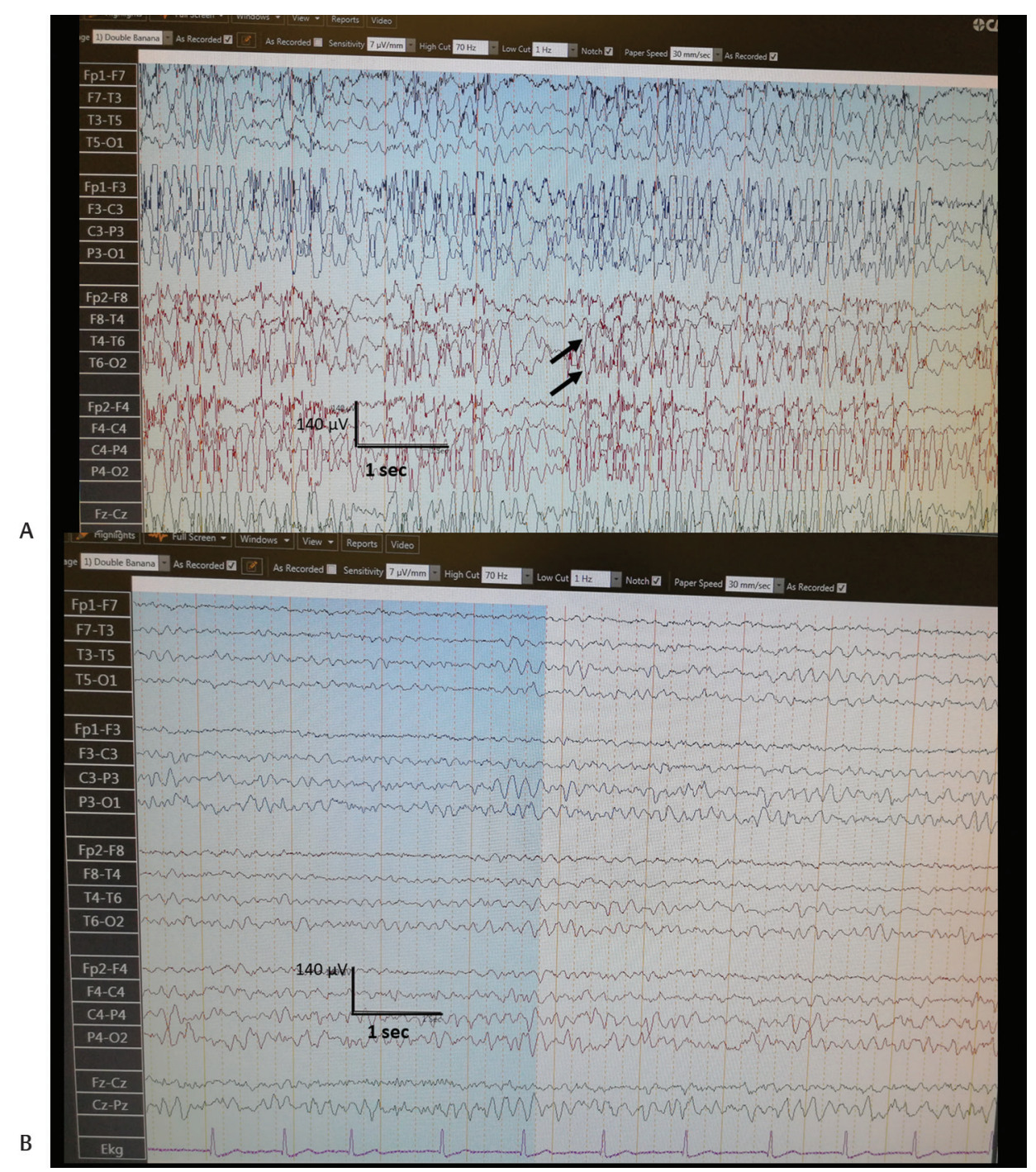

Fig. 2 (A) Routine EEG, performed on the same day of admission, showing generalized discharges of high-voltage spike/polyspike-wave complexes continuing more than half of the EEG recording (arrows). (B) Routine EEG performed the next day showed nearly totally resolution of the epileptiform discharges. EEG, electroencephalography.

\begin{abstract}
Video 2
Neurological examination, recorded the next day of hospitalization on valproic acid $1,000 \mathrm{mg}$, showing totally resolution of myoclonic movements. Online content including video sequences viewable at: https://www. thiemeconnect.com/products/ejournals/html/10.1055/ s-0040-1709348.
\end{abstract}

elicited by cortical stimulation ${ }^{15}$ and the results of the subdural recordings showing ENM-related spikes. ${ }^{4,15}$ In our patient, the synchronous and sustained nature of the myoclonus and insensitivity to volitional control implied that they shared a single-central rhythm generator. Of note, the consciousness of the patient was not disturbed suggesting rather the preservation of the cortical functions. Taken together, in contrast with a cortical origin, rather a subcortical abnormality restricted to the motor pathways was considered in the forefront as the origin of the clinic. As previously mentioned by Palmer et al,,${ }^{14}$ It may be hypothesized that bilateral ischemic lesions in the pons might have produced a localized rhythm generator by creating an irritative focus. However, another possible origin of this clinic may be the ischemic lesions of the thalamus which also have been hypothesized to be the responsible sites from the myoclonic movements in recent studies. ${ }^{16,17}$ Overall, these views can only be hypothetic in the absence of further advanced investigation results (investigations of motor and somatosensory evoked potentials, functional neuroimaging, etc.).

Another important point to be discussed may be the atypical manifestation of status epilepticus in our case. The mortality of status epilepticus is known to be highest in the oldest, and it has been shown that new-onset epilepsy has a higher incidence of status epilepticus in the elderly population..$^{18}$ Although its incidence has not been investigated in the subgroup of myoclonic status 
epilepticus, a mortality rate of approximately $50 \%$ in has been reported in overall patients with status epilepticus those over 80 years of age. ${ }^{19}$ Hence, we also suggest that in presentations of atypical movement disorders in elderly patients, performing EEG may be a vital tool for the proper diagnosis and timely interventions. Beside confusion, sleepiness which may be manifestations of "epileptic amnesia" or prolonged postictal states may be encountered more often in the elderly, rather than motor manifestations. ${ }^{20,21}$ Such that, these features may mask the clinical seizures and lead to underdiagnosis of seizures by the caregivers. ${ }^{20}$ However, in our patient, the clinical presentation was characterized by generalized myoclonus and the patient's cognition was not disturbed which makes this presentation further interesting.

\section{Limitations}

The limitation of our report may be that simultaneous EEG-electromyography (EMG) monitoring was not performed to reveal a silent period before negative myoclonus, demonstrate the association of an EEG spike-time locked to negative myoclonus, and distinguish positive myoclonus. However, it would not be ethical to perform further electrophysiological investigations instead of promptly administration of antiepileptic therapy for status epilepticus. The second limitation was that functional neuroimaging was not conducted which might probably give crucial perspectives regarding the pathophysiology of ictal negative myoclonus. However, this is surely a crucial illustration of an extremely rare etiology of ictal negative myoclonus potentially giving substantial perspectives regarding the underlying mechanisms of positive and negative myoclonus. Future reports of larger case series using multimodal investigations are warranted which may provide substantial contributions regarding the unknown aspects of ictal myoclonus.

\section{Funding}

None.

\section{Conflict of Interest}

None declared.

\section{References}

1 Yu HY, Kwan SY, Lirng JF, Liao KK, Chu YK, Liao SQ. Epileptic negative myoclonus: SPECT, PET, and video/EEG studies and the dramatic effects of levetiracetam. Epilepsy Behav 2009;14(4):687-690
2 Shibasaki $\mathrm{H}$. Pathophysiology of negative myoclonus and asterixis. Adv Neurol 1995;67:199-209

3 Watemberg N, Leitner Y, Fattal-Valevski A, Kramer U. Epileptic negative myoclonus as the presenting seizure type in rolandic epilepsy. Pediatr Neurol 2009;41(1):59-64

4 Rubboli G, Tassinari CA. Negative myoclonus. An overview of its clinical features, pathophysiological mechanisms, and management. Neurophysiol Clin 2006;36(5,6):337-343

5 Tassinari CA, Rubboli G, Gardella E. Negative myoclonus. Clin Neurosci 1995-1996;3(4):209-213

6 Toro C, Hallett M, Rothwell JC, Asselman PT, Marsden CD. Physiology of negative myoclonus. Adv Neurol 1995;67: 211-217

7 Ramsay RE, Rowan AJ, Pryor FM. Special considerations in treating the elderly patient with epilepsy. Neurology 2004; 62(5, Suppl 2):S24-S29

8 Ghosh S, Jehi LE. New-onset epilepsy in the elderly: challenges for the internist. Cleve Clin J Med 2014;81(8):490-498

9 Hauser WA, Annegers JF, Kurland LT. Incidence of epilepsy and unprovoked seizures in Rochester, Minnesota: 1935-1984. Epilepsia 1993;34(3):453-468

10 Pugh MJ, Knoefel JE, Mortensen EM. Amuan ME, Berlowitz DR, Van Cott AC. New-onset epilepsy risk factors in older veterans. J Am Geriatr Soc 2009;57(2):237-242

11 Annegers JF, Hauser WA, Lee JR, Rocca WA. Incidence of acute symptomatic seizures in Rochester, Minnesota, 1935-1984. Epilepsia 1995;36(4):327-333

12 Brodie MJ, Elder AT, Kwan P. Epilepsy in later life. Lancet Neurol 2009;8(11):1019-1030

13 Kilpatrick CJ, Davis SM, Tress BM, Rossiter SC, Hopper JL, Vandendriesen ML. Epileptic seizures in acute stroke. Arch Neurol 1990;47(2):157-160

14 Palmer JB, Tippett DC, Wolf JS. Synchronous positive and negative myoclonus due to pontine hemorrhage. Muscle Nerve 1991;14(2):124-132

15 Rubboli G, Mai R, Meletti S, et al. Negative myoclonus induced by cortical electrical stimulation in epileptic patients. Brain 2006;129(Pt 1) :65-81

16 Bejjani BP, Arnulf I, Vidailhet M, et al. Irregular jerky tremor, myoclonus, and thalamus: a study using low-frequency stimulation. Mov Disord 2000;15(5):919-924

17 Gupta N, Pandey S. Post-thalamic stroke movement disorders: a systematic review. Eur Neurol 2018;79(5,6):303-314

18 Kim DW, Oh J. Comparison of new-onset and persistent epilepsy in the elderly. Acta Neurol Scand 2019;139(4):395-398

19 Leppik IE. Status epilepticus in the elderly. Epilepsia 2018;59(Suppl 2) :140-143

20 Theodore WH. The postictal state: effects of age and underlying brain dysfunction. Epilepsy Behav 2010;19(2):118-120

21 Silveira DC, Jehi L, Chapin J, et al. Seizure semiology and aging. Epilepsy Behav 2011;20(2):375-377 